\title{
Dynamic membrane applications in anaerobic and aerobic digestion for industrial wastewater: a mini review
}

\begin{abstract}
Industrial wastewater treatment using aerobic and anaerobic digestion using membrane bioreactor (MBR) has become popular due to its increasing worldwide application. MBR offers complete separation of solid-liquid by a porous membrane to produce high-quality effluent for potential non-potable water reuse. Dynamic membrane (DM) as alternative to the conventional membrane is suitable for both aerobic and anaerobic digestion. Throughout the years, DM technology has been applied to both systems and the performance was better than that of conventional membrane in terms of filtration capability, easy back washing, and insitu reformation and economically favourable. Production of high-quality effluent with low total solid, high operational stability, treatment of wastewater under extreme conditions such as industrial wastewater has made DM used in aerobic or anaerobic systems significant. Some drawbacks remain, including membrane fouling with excessive layer cake formation made the reactor operation become unstable and time-consuming which also requires high energy for pumping. Dynamic membrane can alleviate these drawbacks using cake layer membrane itself as the filtration devices. In addition, DM uses low-cost materials as the support material, which is a better replacement to the more expensive and high maintenance conventional membranes. Further research is needed to investigate the conditions and characteristics in the dynamic membrane's active layer cake, mechanism and its structure. Identification of the optimal conditions to ensure long-term operation thus improving final effluent without further post-treatment is also needed. In this mini review, in-depth DMs application on aerobic and anaerobic digestion is summarized and evaluated for industrial wastewater treatment.
\end{abstract}

Keyword: Dynamic membrane; Aerobic digestion; Anaerobic digestion; Industrial wastewater; Low-cost membrane; Membrane bioreactor 